\title{
Postoperative hyperlactatemia and serum lactate level trends among heart transplant recipients
}

\author{
Anna Kędziora ${ }^{\text {Corresp., } 1}{ }^{1}$, Karol Wierzbicki ${ }^{1}$, Jacek Piątek ${ }^{1}{ }$, Hubert Hymczak ${ }^{2}$, Izabela Górkiewicz-Kot ${ }^{1}$, Irena \\ Milaniak $^{1,3}$, Paulina Tomsia ${ }^{1}$, Dorota Sobczyk ${ }^{4}$, Rafał Drwiła ${ }^{2}$, Bogusław Kapelak ${ }^{1}$ \\ 1 Department of Cardiovascular Surgery and Transplantology, John Paul II Hospital; Institute of Cardiology, Jagiellonian University Medical College, Krakow, \\ Poland \\ 2 Department of Anesthesiology and Intensive Care, John Paul II Hospital, Krakow, Poland \\ 3 Wydział Lekarski i Nauk o Zdrowiu, Krakowska Akademia im. Andrzeja Frycza-Modrzewskiego, Kraków, Polska \\ 4 Department of Cardiac and Vascular Diseases, John Paul II Hospital; Institute of Cardiology, Jagiellonian University Medical College, Krakow, Poland \\ Corresponding Author: Anna Kędziora \\ Email address: anna.kedziora@doctoral.uj.edu.pl
}

Background. Advanced heart failure (HF), that affects $10 \%$ of the HF population, is associated with high mortality rate, meeting $50 \%$ at 1-year from diagnosis. For these individuals, heart transplantation (HTX) remains the ultimate and the gold-standard treatment option. Serum lactate level measurements has been proven useful for determining the outcome following other cardiac surgeries and among critically ill patients. Increased serum lactate levels are expected following HTX, however, no detailed analysis has been yet performed in this population. The research aims to estimate the prevalence of hyperlactatemia and describe early postoperative serum lactate level trends among heart transplant recipients.

Materials and Methods. Forty six consecutive patients, who underwent HTX between 2010 and 2015, were enrolled into the retrospective analysis. Serum lactate level measurements within first 48 hours post-HTX were obtained every 6 hours from routinely conducted arterial blood gas analyses. The threshold for hyperlactatemia was considered at $>1.6 \mathrm{mmol} / \mathrm{L}$, according to upper limit of normal, based on internal laboratory standardization. The highest observed measurement within the observation, regardless of the time point of observation was determined for each patient individually and was appointed as Peak Value.

Results. Consecutively measured serum lactate levels differed in time $(p=0.000)$, with the initial increase and subsequent decrease of the values ( $4.3 \mathrm{vs} 1.9 \mathrm{mmol} / \mathrm{l} ; \mathrm{p}=0.000)$. The increase from the baseline level to the Peak Value was statistically significant ( $4.3 \mathrm{vs} 7.0 \mathrm{mmol} / \mathrm{l} ; \mathrm{p}=0.000$ ). Various serum lactate level trends were identified, with one or more hyperlactatemia episodes. Eventually, $50 \%$ of the individuals had normal serum lactate levels at the end of the study, and hyperlactatemia was observed in the other half.

Conclusions. Throughout the observation, all of the patients experienced at least one episode of hyperlactatemia, with the median Peak Value of $7.0(4.5-8.4) \mathrm{mmol} / \mathrm{L}$. Various serum lactate level trends can be identified in post-HTX patients. Further research is required to determine the clinical usefulness of newly reported serum lactate level trends among heart transplant recipients. 
1 Postoperative hyperlactatemia and serum lactate level trends among heart transplant

2 recipients

3

4 Anna Kędziora $^{1}$, Karol Wierzbicki ${ }^{1}$, Jacek Piątek ${ }^{1}$, Hubert Hymczak ${ }^{2}$, Izabela Górkiewicz-Kot ${ }^{1}$,

5 Irena Milaniak ${ }^{1}$, Paulina Tomsia ${ }^{1}$, Dorota Sobczyk ${ }^{3}$, Rafał Drwiła ${ }^{2}$, Bogusław Kapelak ${ }^{1}$

6

7 1. Department of Cardiovascular Surgery and Transplantology, John Paul II Hospital;

8 Institute of Cardiology, Jagiellonian University Medical College, Krakow, Poland

9

2. Department of Anesthesiology and Intensive Care, John Paul II Hospital, Krakow, Poland

3. Department of Cardiac and Vascular Diseases, John Paul II Hospital; Institute of Cardiology, Jagiellonian University Medical College, Krakow, Poland

The authors declare no conflict of interest

14 The study was funded by the grant from Jagiellonian University Medical College 


\section{Abstract}

23 Background. Advanced heart failure (HF), that affects $10 \%$ of the HF population, is associated with high mortality rate, meeting $50 \%$ at 1 -year from diagnosis. For these individuals, heart transplantation (HTX) remains the ultimate and the gold-standard treatment option. Nevertheless, despite the total of approximately 5,000 HTX performed worldwide annually, there is a gap in scientific evidence in terms of early postoperative management for heart transplant recipients. Despite the fact, that it is justified to assume that in post-HTX patients serum lactate levels will be elevated and that they can presumably be used to determine the outcome, the exploration of standard threshold for hyperlactatemia in post-HTX patients has never been considered.

Materials and Methods. Forty six consecutive patients, who underwent HTX between 2010 and 2015, were enrolled into the retrospective analysis. Serum lactate level measurements within first 48 hours post-HTX were obtained every 6 six hours from routinely conducted arterial blood gas analyses. The threshold for hyperlactatemia was considered at $>1.6 \mathrm{mmol} / \mathrm{L}$, based on internal laboratory standardization. The Peak Value represents the highest observed measurement within the observation, regardless of the time point of observation, and was determined for each patient

37 individually.

38 Results. Consecutive serum lactate level measurements differed in time $(p=0.000)$. The increase from the baseline level to the Peak Value was statistically significant $(\mathrm{p}=0.000)$, and similarly was

40 the decrease throughout the observation $(\mathrm{p}=0.000)$. Various serum lactate level trends were 41 identified, with different numbers of hyperlactatemia and normalization episodes. Eventually, 50\% 42 of the individuals had normal serum lactate levels at the end of the study, and hyperlactatemia was 
43 observed in the other half. One-third of the patients with the lowest baseline serum lactate levels 44 experienced sequent hyperlactatemia episode and patients who entered the study with the Peak

45 Value as the baseline observation, completed the analysis with lowest values after a considerable 46 curve slope within first 12 hours of observation.

47 Conclusions. Throughout the observation, all of the patients experienced at least one episode of 48 hyperlactatemia, with the median Peak Value of $7.0(4.5$ - 8.4) $\mathrm{mmol} / \mathrm{L}$. Various serum lactate 49 level trends can be identified in post-HTX patients. Further research is required to determine the 50 clinical usefulness of newly reported serum lactate level trends among heart transplant recipients.

51

52

53

54

55

56

57

58

59

60

61

62 
Introduction

66

67

Heart failure (HF), with the current prevalence of $2.5 \%$, is a major public health problem associated with significant hospital admission rates, mortality, and health care costs (Benjamin et al. 2017). The age-adjusted rate for heart failure-related deaths has been steadily increasing since 2012 , reaching 84.0 deaths per 100,000 standard population in 2014 (Ni and $\mathrm{Xu}$ 2015). Advanced HF, that affects $10 \%$ of the HF patients, is associated with even higher mortality rate, meeting $50 \%$ at 1-year from diagnosis (Lund 2018). For these individuals, heart transplantation (HTX) remains the ultimate and the gold-standard treatment option (Ponikowski et al. 2016). Nevertheless, despite the total of approximately 5,000 HTX performed worldwide annually (Khush et al. 2018), there is still a gap in scientific evidence in terms of early postoperative management for heart transplant recipients. The immunosuppression, anti-infective prophylaxis, and pulmonary hypertension treatment have been widely discussed in this group of patients (Costanzo et al. 2010), however, only few postoperative biochemical measurements have been thoroughly analyzed so far (Wierzbicki et al. 2014; Madershahian et al. 2008).

Under basal conditions, the lactate is produced by all tissues and reutilized at a nearly constant rate by the liver. Normally, lactate concentration in the extracellular fluid is about $1 \mathrm{mmol} / \mathrm{l}$. The diagnostic lower and upper limits of normal slightly vary (from 0.3 to $2.0 \mathrm{mmol} / \mathrm{l}$ ) according to the specimen (arterial or venous blood) and method used (Toffaletti 1991). The overproduction and, therefore, increased levels can be observed during tissue oxygen deficiency as a result of the circulatory shock and perfusion failure (Adeva-Andany et al. 2014). The potential usefulness of 
85 lactate measurements as an indicator of the shock severity has been has been first evaluated in 861964 (Broder and Weil 1964). Since then, numerous studies have reported data for measurements 87 taken in various acute clinical settings and, throughout time, cut-off values for therapeutic 88 management have evolved (Zhou et al. 2017).

89 Similarly, hyperlactatemia assessment has been proven useful in determining the outcome in some 90 cardiosurgical procedures, as it reflects the problem of postoperative hypoperfusion (Hajjar et al. 91 2013; Andersen et al. 2015; Lopez-Delgado et al. 2015). Following HTX, increased serum lactate 92 levels are expected, as prolonged cardiopulmonary bypass (CPB) time and compromised

93 hemodynamic function within early postoperative hours are commonly observed. However, 94 postoperative serum lactate level trends and clinical usefulness of lactate measurements have never 95 been analyzed before among post-HTX patients. Moreover, currently available guidelines for the 96 care of heart transplant recipients are mostly based on the consensus of opinion of the experts 97 (level of evidence C) (Costanzo et al. 2010), which indicates the gap in scientific evidence.

98 Therefore, the presented research aims to estimate the actual prevalence of hyperlactatemia and 99 describe early postoperative serum lactate level trends among heart transplant recipients.

\section{Materials and methods}

Forty six consecutive patients, who underwent HTX in the Department of Cardiovascular Surgery and Transplantology between 2010 and 2015, were enrolled into the retrospective analysis. Serum lactate level measurements within first 48 hours post-HTX were obtained from arterial blood gas (ABG) analyses, that were routinely conducted every 6 hours. The mean age of the study group 
106 was $48.7 \pm 11.7$ years. The majority of the patients were males $(89.1 \%)$ and were qualified for

107 HTX due to dilated cardiomyopathy (73.9\%) (Table 1).

108 Consecutive serum lactate level measurements, that were taken every 6 hours, were appointed as

$109 L a c$, with the following number as the time point (hour) of observation. The baseline measurement

110 was defined as the observation taken at Intensive Care Unit (ICU) admission (Lac 0). The end of

111 the study measurement was defined as the observation taken at the $48^{\text {th }}$ hour post-HTX (Lac 48).

112 The highest observed measurement within the observation, regardless of the time point of

113 observation was determined for each patient individually and was presented as the Peak Value.

114 The threshold for hyperlactatemia $(>1.6 \mathrm{mmol} / \mathrm{L})$ was defined by the upper limit value of the

115 normal range for serum lactate concentration $(0.5-1.6 \mathrm{mmol} / \mathrm{l})$, based on internal laboratory

116 standardization for ABG analysis. ABG analysis was performed with an automated electrode

117 analyzer (“Arterial Blood Gases - Clinical Methods - NCBI Bookshelf” n.d.).

118 The study obtained the Jagiellonian University Bioethics Committee approval

119 (122.6120.74.2017). According to the approval, verbal consent was obtained from all living 120 patients.

121 Statistical analysis

122 Statistical analysis was performed with IBM $^{\circledR}$ SPSS ${ }^{\circledR}$ Statistics 25 . Normal distribution was tested

123 using the Shapiro-Wilk test. Continuous variables are presented as means and standard deviation

$124( \pm \mathrm{SD})$ or medians and interquartile ranges $(\mathrm{IQR})$. For categorical variables, numbers and

125 proportions are reported. Non-parametric tests for independent (Kruskal-Wallis test) or dependent

126 samples (Wilcoxon signed-rank test, Friedman test), where appropriate, were calculated to

127 determine the differences between observations. 


\section{Results}

129 The values of the consecutive serum lactate level measurements differed in time $(p=0.000$;

130 Friedman test) and initial increase with subsequent decrease throughout the study was observed.

131 The increase from the baseline level (Lac 0) to the highest observed value (Peak Value) was

132 statistically significant ( $\mathrm{p}=0.000$; Wilcoxon signed-rank test), and similarly was the decrease

133 throughout the observation (from Lac 0 to Lac 48; $\mathrm{p}=0.000$; Wilcoxon signed-rank test) (Figure 134 1).

135 In the detailed analysis, various serum lactate level trends were identified. In the majority of the 136 patients (63\%) serum lactate level normalization was achieved, however, one-third (33\%) of the 137 study group experienced sequent episode of hyperlactatemia after first normalization. Eventually, $13850 \%$ of the individuals had normal serum lactate levels at the end of the study (Lac 48), and 139 hyperlactatemia was observed in the other half (Figure 2).

140 Further calculations determined the association between the baseline serum lactate level ( $\operatorname{Lac} 0$ as 141 normal or hyperlactatemia or peak lactates) and the highest observed serum lactate level (Peak

142 Value $)(\mathrm{p}=0.06$; Kruskal-Wallis test; Figure 3). No such association was found for the end of the 143 study serum lactate level (Lac 48 as normal or hyperlactatemia) $(\mathrm{p}=0.263$; Kruskal-Wallis test; 144 Figure 4).

Discussion

147 Under anaerobic conditions, cellular metabolism converts pyruvate to lactate. Therefore, serum

148 lactate levels relate to the oxygen debt and correspond with the severity of tissue hypoperfusion 149 (Adeva-Andany et al. 2014; Hoshino, Kinoshita, and Ono 2018). The normal plasma lactate 
150 concentration for $\mathrm{ABG}$ is reported from 0.3 to $1.6 \mathrm{mmol} / \mathrm{L}$ (Broder and Weil 1964), whereas lactic 151 acidosis is generally defined with a threshold of greater than $4 \mathrm{mmol} / \mathrm{L}$, even in the absence of 152 overt acidemia. Type A lactic acidosis, that is considered to be a medical emergency and is 153 associated with poor prognosis when left untreated, is usually caused by significant tissue 154 hypoperfusion (Andersen et al. 2013).

155 Hyperlactatemia is a common phenomenon following cardiac surgeries, as it can result from not only hypoxic but also nonhypoxic causes such as drug therapy, cardioplegia, hypothermia, and CPB. Previous studies among cardiosurgical patients, comparing survivors vs non-survivors, have indicated that that higher lactate levels upon ICU admission $(2-3 \mathrm{mmol} / \mathrm{l})$, or within early postoperative hours $(3-4 \mathrm{mmol} / \mathrm{l})$, are associated with increased perioperative risk and prolonged hospital stay (Hajjar et al. 2013; Lopez-Delgado et al. 2015; Andersen et al. 2015).

Nevertheless, to date only two studies, concerning the issue of postoperative hyperlactatemia among heart transplant recipients have been published. Both of the researches were single center studies that evaluated solely whether the patient has reached the threshold, set by the authors, at any time point throughout the observation. The extreme hyperlactatemia was defined by Hsu et al. as the serum lactate level greater than $15 \mathrm{mmol} / \mathrm{L}$ and occurred in 3 patients at the ICU admission and in 9 others within early postoperative hours (12/58 patients). In spite of the further serum lactate level decrease below $4 \mathrm{mmol} / \mathrm{L}$, one-third of the analyzed hyperlactatemia subgroup deceased within the hospitalization, and the others within nearly 5 years post-HTX (Hsu et al. 2015). In another study, the cut-off level of $4 \mathrm{mmol} / \mathrm{L}$ was set according to the guidelines for severe sepsis and septic shock treatment. Based on this estimation, elevated serum lactate levels were observed among 59.2\% of post-HTX patients, with higher occurrence of postoperative pulmonary complications within this subgroup. As no in-hospital deaths were reported, no testing was 
173 performed to determine the impact on early mortality (Hoshino, Kinoshita, and Ono 2018).

174 Nevertheless, the detailed exploration of the cut-off value, appointed by the upper limit of normal

175 for serum lactate concentration, has never been presented in post-HTX patients before.

176 In the presented research, hyperlactatemia was considered with the of $>1.6 \mathrm{mmol} / \mathrm{l}$, same as it is

177 for other cardiac surgeries and non-surgical patients in our center. Despite the initially emerging

178 pattern with early peak of the lactate values, that was similar to other post-HTX lactate analyses

179 (Hsu et al. 2015; Hoshino, Kinoshita, and Ono 2018), more detailed evaluation revealed various

180 serum lactate level trends. The interpretation of the initial results indicates the increase to the

181 highest serum lactate level within first 6 postoperative hours with subsequent gradual decrease

182 (Figure 1). However, close case-by-case investigation provided more rigorous outcome, showing 183 differences in the number of hyperlactatemia episodes and time intervals in between (Figure 2).

184 Serum lactate level trends significantly differed between subgroups assigned based on the baseline 185 serum lactate level (Lac 0 as normal or hyperlactatemia or peak lactates). The highest observed measurement (Peak Value) varied between the subgroups. Although the lowest Peak Value was 187 seen in the normal group, two serum lactate level spikes can be identified $\left(12^{\text {th }}\right.$ and $30^{\text {th }}$ hour of observation). Based on the metadata, in half of these patients Peak Value was noted at $12^{\text {th }}$ hour 189 post-surgery, and in the other half later within observation. Moreover, one-third of these individuals actually experienced sequent hyperlactatemia episode (Figure 3). On the other hand, 191 patients who entered the study with their highest serum lactate levels (Lac 0 as peak lactates), completed the analysis with lowest values after a considerable curve slope within first 12 hours of observation (Figure 3). Similar curve slope difference was noted among the subgroups assigned 
195 at $18^{\text {th }}$ hour of observation, resulting in elevated serum lactate levels at the end of the study in the 196 hyperlactatemia subgroup (Figure 4).

197 The planned extension of this study includes the search for pre- and intraoperative factors

198 determining various serum lactate level trends, as well as for the association between various 199 serum lactate level trends and the postoperative management and course, with the special focus on 200 the hemodynamic function of the transplanted heart.

\section{Conclusions}

203

204

205

206

207

208

209

210

211

212

213

214

215

Throughout the observation, all of the patients experienced at least one episode of hyperlactatemia, with the median peak value of $7.0(4.5-8.4) \mathrm{mmol} / \mathrm{L}$. Various serum lactate level trends can be identified in post-HTX patients, with the greatest differences in terms of the ICU admission measurement, and the highest observed value. Further research is required to determine the clinical usefulness of newly reported serum lactate level trends among heart transplant recipients.

\section{References}

Adeva-Andany, M., M. López-Ojén, R. Funcasta-Calderón, E. Ameneiros-Rodríguez, C. Donapetry-García, M. Vila-Altesor, and J. Rodríguez-Seijas. 2014. “Comprehensive Review on Lactate Metabolism in Human Health.” Mitochondrion 17 (July): 76-100. https://doi.org/10.1016/j.mito.2014.05.007.

Andersen, Lars W., Mathias J. Holmberg, Michelle Doherty, Kamal Khabbaz, Adam Lerner, Katherine M. Berg, and Michael W. Donnino. 2015. "Postoperative Lactate Levels and 
216

217

218

219

220

221

222

223

224

225

226

227

228

229

230

231

232

233

234

235

236

237

Hospital Length of Stay After Cardiac Surgery.” Journal of Cardiothoracic and Vascular Anesthesia 29 (6): 1454-60. https://doi.org/10.1053/j.jvca.2015.06.007.

Andersen, Lars W., Julie Mackenhauer, Jonathan C. Roberts, Katherine M. Berg, Michael N. Cocchi, and Michael W. Donnino. 2013. "Etiology and Therapeutic Approach to Elevated Lactate.” Mayo Clinic Proceedings 88 (10): 1127. https://doi.org/10.1016/J.MAYOCP.2013.06.012.

“Arterial Blood Gases - Clinical Methods - NCBI Bookshelf." n.d. Accessed November 11, 2019. https://www.ncbi.nlm.nih.gov/books/NBK371/.

Benjamin, Emelia J, Michael J Blaha, Stephanie E Chiuve, Mary Cushman, Sandeep R Das, Rajat Deo, Sarah D de Ferranti, et al. 2017. "Heart Disease and Stroke Statistics-2017 Update: A Report From the American Heart Association.” Circulation 135 (10): e146-603. https://doi.org/10.1161/CIR.0000000000000485.

Broder, George, and Max H. Weil. 1964. "Excess Lactate: An Index of Reversibility of Shock in Human Patients.” Science 143 (3613): 1457-59. https://doi.org/10.1126/science.143.3613.1457.

Costanzo, Maria Rosa, Maria Rosa Costanzo, Anne Dipchand, Randall Starling, Allen Anderson, Michael Chan, Shashank Desai, et al. 2010. “The International Society of Heart and Lung Transplantation Guidelines for the Care of Heart Transplant Recipients.” The Journal of Heart and Lung Transplantation 29 (8): 914-56. https://doi.org/10.1016/j.healun.2010.05.034.

Hajjar, Ludhmila A., Juliano P. Almeida, Julia T. Fukushima, Andrew Rhodes, Jean-Louis Vincent, Eduardo A. Osawa, and Filomena R.B.G. Galas. 2013. "High Lactate Levels Are 
238

239

240

241

242

243

Predictors of Major Complications after Cardiac Surgery.” The Journal of Thoracic and Cardiovascular Surgery 146 (2): 455-60. https://doi.org/10.1016/j.jtcvs.2013.02.003.

Hoshino, Yasuhiro, Osamu Kinoshita, and Minoru Ono. 2018. “The Incidence, Risk Factors, and Outcomes of Hyperlactatemia after Heart Transplantation.” International Heart Journal 59 (1): 81-86. https://doi.org/10.1536/ihj.17-146.

Hsu, Y.C., C.H. Hsu, G.S. Huang, C.C. Lu, Z.F. Wu, Y.T. Tsai, C.Y. Lin, Y.C. Lin, C.S. Tsai, and T.C. Lin. 2015. “Extreme Hyperlactatemia After Heart Transplantation: One Center's Experience." Transplantation Proceedings 47 (6): 1945-48. https://doi.org/10.1016/j.transproceed.2015.02.027.

Khush, Kiran K., Wida S. Cherikh, Daniel C. Chambers, Samuel Goldfarb, Don Hayes, Anna Y. Kucheryavaya, Bronwyn J. Levvey, Bruno Meiser, Joseph W. Rossano, and Josef Stehlik. 2018. "The International Thoracic Organ Transplant Registry of the International Society for Heart and Lung Transplantation: Thirty-Fifth Adult Heart Transplantation Report2018; Focus Theme: Multiorgan Transplantation.” The Journal of Heart and Lung Transplantation 37 (10): 1155-68. https://doi.org/10.1016/j.healun.2018.07.022.

Lopez-Delgado, Juan C., Francisco Esteve, Casimiro Javierre, Herminia Torrado, David Rodriguez-Castro, Maria L. Carrio, Elisabet Farrero, Konstantina Skaltsa, Rafael Mañez, and Josep L. Ventura. 2015. "Evaluation of Serial Arterial Lactate Levels as a Predictor of Hospital and Long-Term Mortality in Patients After Cardiac Surgery." Journal of Cardiothoracic and Vascular Anesthesia 29 (6): 1441-53. https://doi.org/10.1053/j.jvca.2015.04.024.

Lund, Lars H. 2018. “Optimizing Outcomes after Heart Transplantation.” European Journal of 
Heart Failure 20 (2): 395-97. https://doi.org/10.1002/ejhf.1026.

261

262

263

264

265

266

267

268

269

270

271

272

273

274

275

276

277

278

279

280

Madershahian, Navid, Thorsten Wittwer, Justus Strauch, Jens Wippermann, Parwis Rahmanian, Ulrich F.W. Franke, and Thorsten Wahlers. 2008. "Kinetic of Procalcitonin in the Early Postoperative Course Following Heart Transplantation." Journal of Cardiac Surgery 23 (5): 468-73. https://doi.org/10.1111/j.1540-8191.2008.00625.x.

Ni, Hanyu, and Jiaquan Xu. 2015. "Recent Trends in Heart Failure-Related Mortality: United States, 2000-2014." NCHS Data Brief, no. 231 (December): 1-8. http://www.ncbi.nlm.nih.gov/pubmed/26727546.

Ponikowski, Piotr, Adriaan A. Voors, Stefan D. Anker, Héctor Bueno, John G. F. Cleland, Andrew J. S. Coats, Volkmar Falk, et al. 2016. "2016 ESC Guidelines for the Diagnosis and Treatment of Acute and Chronic Heart Failure.” European Heart Journal 37 (27): 2129_ 2200. https://doi.org/10.1093/eurheartj/ehw128.

Toffaletti, J G. 1991. “Blood Lactate: Biochemistry, Laboratory Methods, and Clinical Interpretation.” Critical Reviews in Clinical Laboratory Sciences 28 (4): 253-68. https://doi.org/10.3109/10408369109106865.

Wierzbicki, Karol, Maciej Bochenek, Anna Kędziora, Krzysztof Sojecki, Dorota CiołczykWierzbicka, Piotr Węgrzyn, Rafał Drwiła, Bogusław Kapelak, Irena Milaniak, and Jerzy Sadowski. 2014. "HEART AND LUNG FAILURE, TRANSPLANTOLOGY Does the 
282

283

284

285

286

287

288

289

290

291

292

293

294

295

296

297

298

299

300

301

302
'Hyperlactatemia' and the Role of Infection.” American Journal of Emergency Medicine.

W.B. Saunders. https://doi.org/10.1016/j.ajem.2017.03.029.

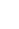

'H


303 Serum lactate level medians differ between baseline (Lac 0) and peak time point (Peak Value):

$304 \mathrm{p}=0.000$; Wilcoxon signed-rank test

305 Data for serum lactate levels are presented as medians (IQR)

306 Lac X-consecutive serum lactate measurements with the following number as the time point

307 (hour) of observation

308 Peak Value - the highest observed serum lactate level within the observation, regardless of the 309 time point

310

311 Figure 2. Study flow chart

312 Data for time intervals and serum lactate levels are presented as medians (IQR)

313 The number of patients at each point is followed by the \% based on the total number of patients 314 in the study

315 Lac X-consecutive serum lactate measurements with the following number as the time point 316 (hour) of observation

317 Peak Value - the highest observed serum lactate level within the observation, regardless of the 318 time point

Figure 3. Serum lactate level trends within 48 hours observation by baseline serum lactate level

322 Baseline (Lac 0) serum lactate level distributions differ among the groups: $p=0.000$; Kruskal323 Wallis test

324 Peak (Peak Value) serum lactate level distributions differ among the groups: $\mathrm{p}=0.06$; Kruskal325 Wallis test

326 Data for serum lactate levels are presented as medians (IQR)

327 Lac X-consecutive serum lactate measurements with the following number as the time point 328 (hour) of observation

329 Peak Value - the highest observed serum lactate level within the observation, regardless of the 330 time point

Figure 4. Serum lactate level trends within 48 hours observation by end of the study serum lactate level

334 End of the study (Lac 48) serum lactate level distributions differ among the groups: $p=0.000$;

335 Kruskal-Wallis test 
336 Data for serum lactate levels are presented as medians (IQR)

337 Lac X-consecutive serum lactate measurements with the following number as the time point 338 (hour) of observation

339 Peak Value - the highest observed serum lactate level within the observation, regardless of the 340 time point

341

342

343

344 


\section{Figure 1}

Figure 1. Serum lactate level trends within 48 hours observation

Serum lactate level distributions differ in time: $\chi^{2}(2)=169.1 ; p=0.000$; Friedman test

Serum lactate level medians differ between baseline ( $\operatorname{Lac} 0)$ and end of the study ( $\operatorname{Lac} 48): p=0.000$;

Wilcoxon signed-rank test

Serum lactate level medians differ between baseline ( $\operatorname{Lac} 0)$ and peak time point (Peak Value): $p=0.000$;

Wilcoxon signed-rank test

Data for serum lactate levels are presented as medians (IQR)

Lac $X$ - consecutive serum lactate measurements with the following number as the time point (hour) of observation

Peak Value - the highest observed serum lactate level within the observation, regardless of the time point 


\section{MEDIAN SERUM LACTATE LEVELS WITHIN 48 HOURS OBSERVATION}

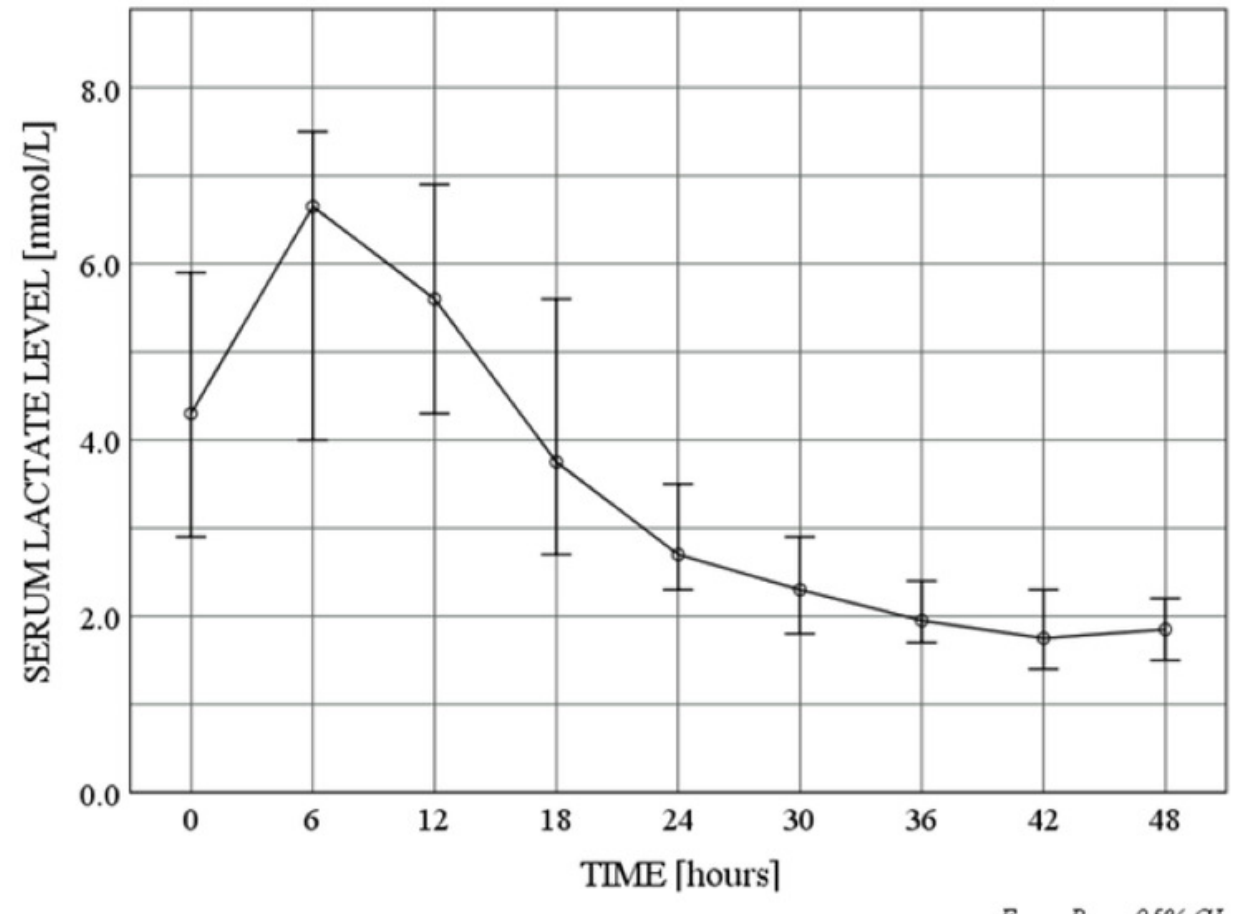

\begin{tabular}{|l|c|}
\hline $\begin{array}{c}\text { OBSERVATION } \\
\text { TIME POINT }\end{array}$ & $\begin{array}{c}\text { SERUM LACTATE } \\
\text { LEVEL [mmol/L] }\end{array}$ \\
\hline Lac 0 & $4.3(2.3-6.4)$ \\
\hline Lac 6 & $6.7(3.3-8.0)$ \\
\hline Lac 12 & $5.6(3.7-7.6)$ \\
\hline Lac 18 & $3.8(2.3-6.1)$ \\
\hline Lac 24 & $2.7(1.9-4.0)$ \\
\hline Lac 30 & $2.3(1.6-3.1)$ \\
\hline Lac 36 & $2.0(1.6-2.5)$ \\
\hline Lac 42 & $1.8(1.3-2.4)$ \\
\hline Lac 48 & $1.9(1.3-2.3)$ \\
\hline Peak Value & $7.0(4.5-8.4)$ \\
\hline
\end{tabular}

Error Bars: $95 \% \mathrm{CI}$ 
Figure 2

Figure 2. Study flow chart

Data for time intervals and serum lactate levels are presented as medians (IQR)

The number of patients at each point is followed by the \% based on the total number of patients in the study

Lac $X$ - consecutive serum lactate measurements with the following number as the time point (hour) of observation

Peak Value - the highest observed serum lactate level within the observation, regardless of the time point
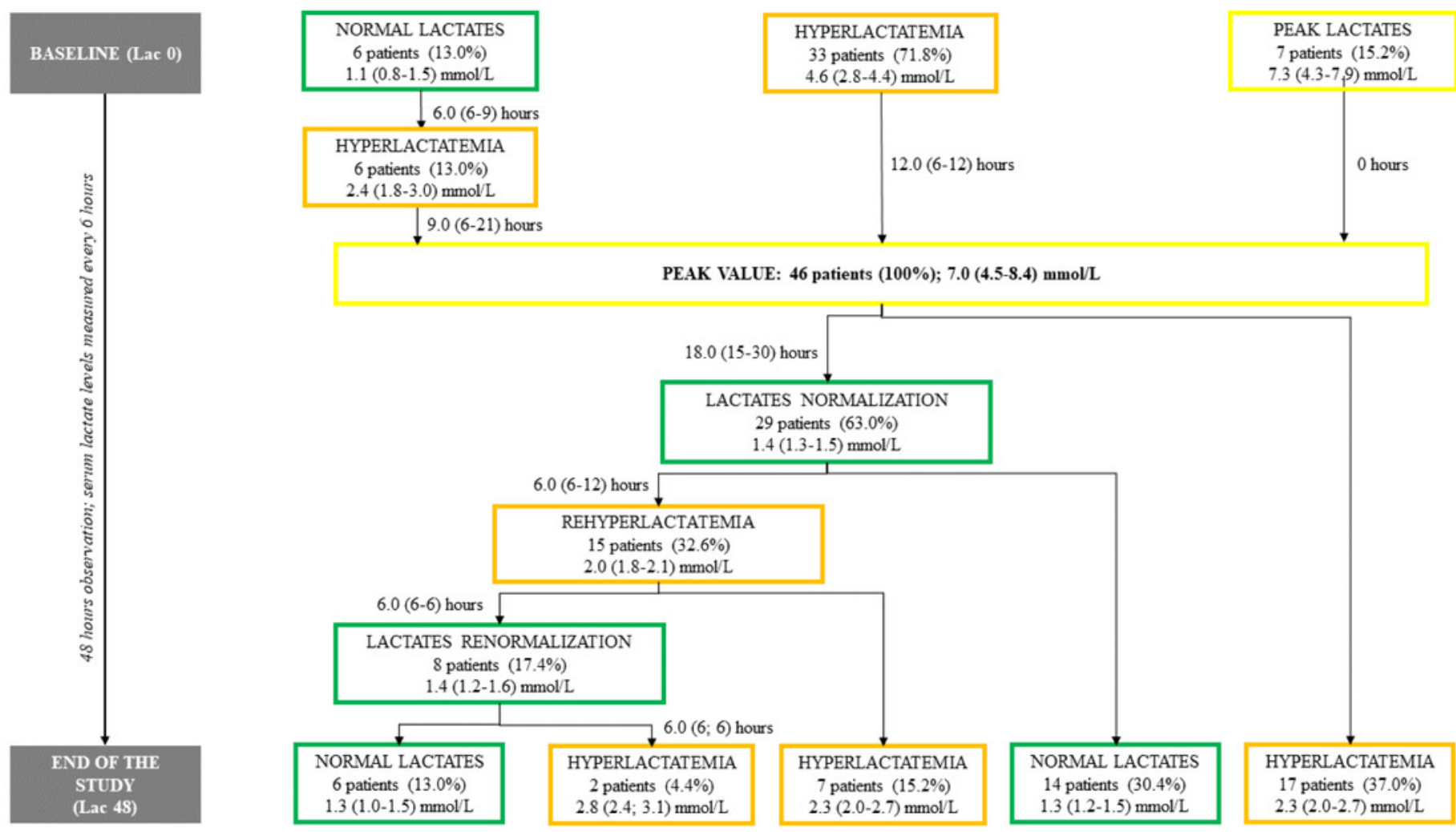
Figure 3

Figure 3. Serum lactate level trends within 48 hours observation by baseline serum lactate level

Baseline (Lac 0) serum lactate level distributions differ among the groups: $p=0.000$; Kruskal-Wallis test Peak (Peak Value) serum lactate level distributions differ among the groups: $p=0.06$; Kruskal-Wallis test Data for serum lactate levels are presented as medians (IQR)

Lac $X$ - consecutive serum lactate measurements with the following number as the time point (hour) of observation

Peak Value - the highest observed serum lactate level within the observation, regardless of the time point

\section{MEDIAN SERUM LACTATE LEVELS WITHIN 48 HOURS OBSERVATION} BY BASELINE SERUM LACTATE LEVEL

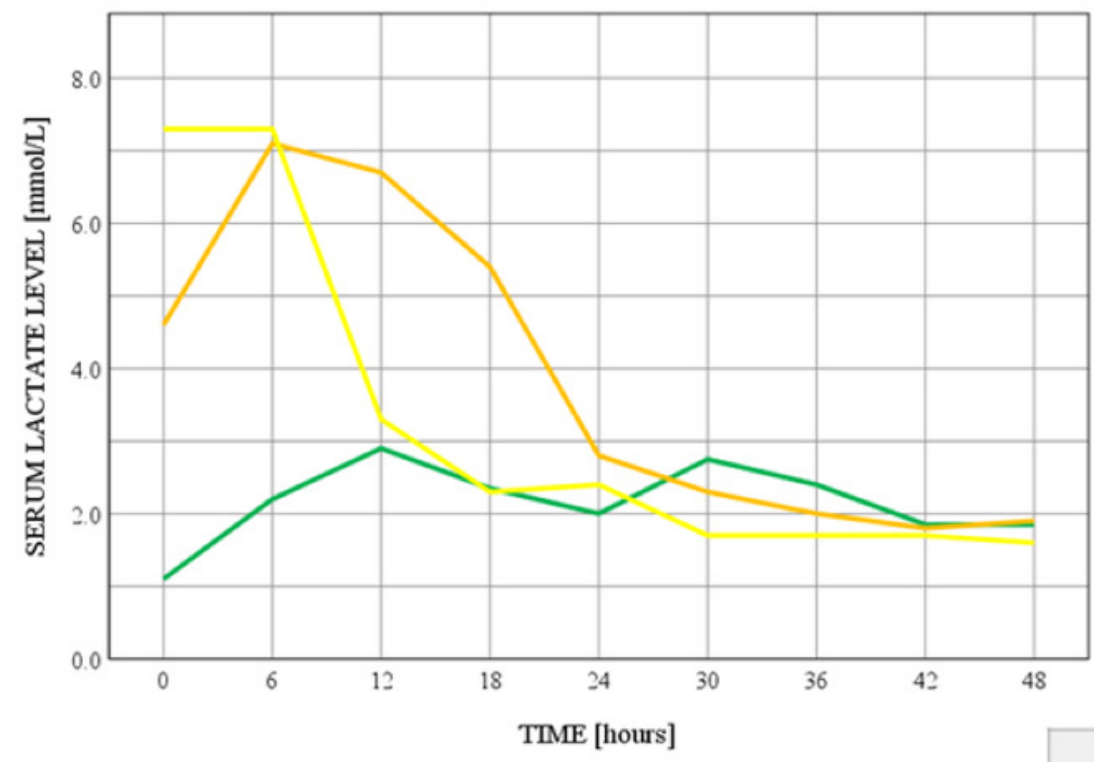

\begin{tabular}{|c|c|c|}
\hline BASELINE & $\begin{array}{l}\text { OBSERVATION } \\
\text { TIME POINT }\end{array}$ & $\begin{array}{l}\text { SERUM LACTATE } \\
\text { LEVEL [mmol/L] }\end{array}$ \\
\hline \multirow{3}{*}{ NORMAL } & Lac 0 & $1.1(0.8-1.5)$ \\
\hline & Lac 48 & $1.9(1.3-2.5)$ \\
\hline & Peak Value & $3.8(2.9-4.5)$ \\
\hline \multirow{3}{*}{ HYPERLACTATEMLA } & Lac 0 & $4.6(2.8-6.4)$ \\
\hline & Lac 48 & $1.9(1.4-2.5)$ \\
\hline & Peak Value & $7.6(5.3-9.0)$ \\
\hline \multirow{3}{*}{ PEAK LACTATES } & Lac 0 & $7.3(4.3-7.9)$ \\
\hline & Lac 48 & $1.6(1.3-2.2)$ \\
\hline & Peak Value & $7.3(4.3-7.9)$ \\
\hline
\end{tabular}

BASELINE SERUM LACTATE LEVEL (Lac 0)

- NORMAL

- HYPERLACTATEMAA

- PEAK LACTATES 


\section{Figure 4}

Figure 4. Serum lactate level trends within 48 hours observation by end of the study serum lactate level

End of the study (Lac 48) serum lactate level distributions differ among the groups: $p=0.000$; Kruskal-Wallis test

Data for serum lactate levels are presented as medians (IQR)

Lac $X$ - consecutive serum lactate measurements with the following number as the time point (hour) of observation

Peak Value - the highest observed serum lactate level within the observation, regardless of the time point

\section{MEDIAN SERUM LACTATE LEVELS WITHIN 48 HOURS OBSERVATION} BY END OF THE STUDY SERUM LACTATE LEVEL

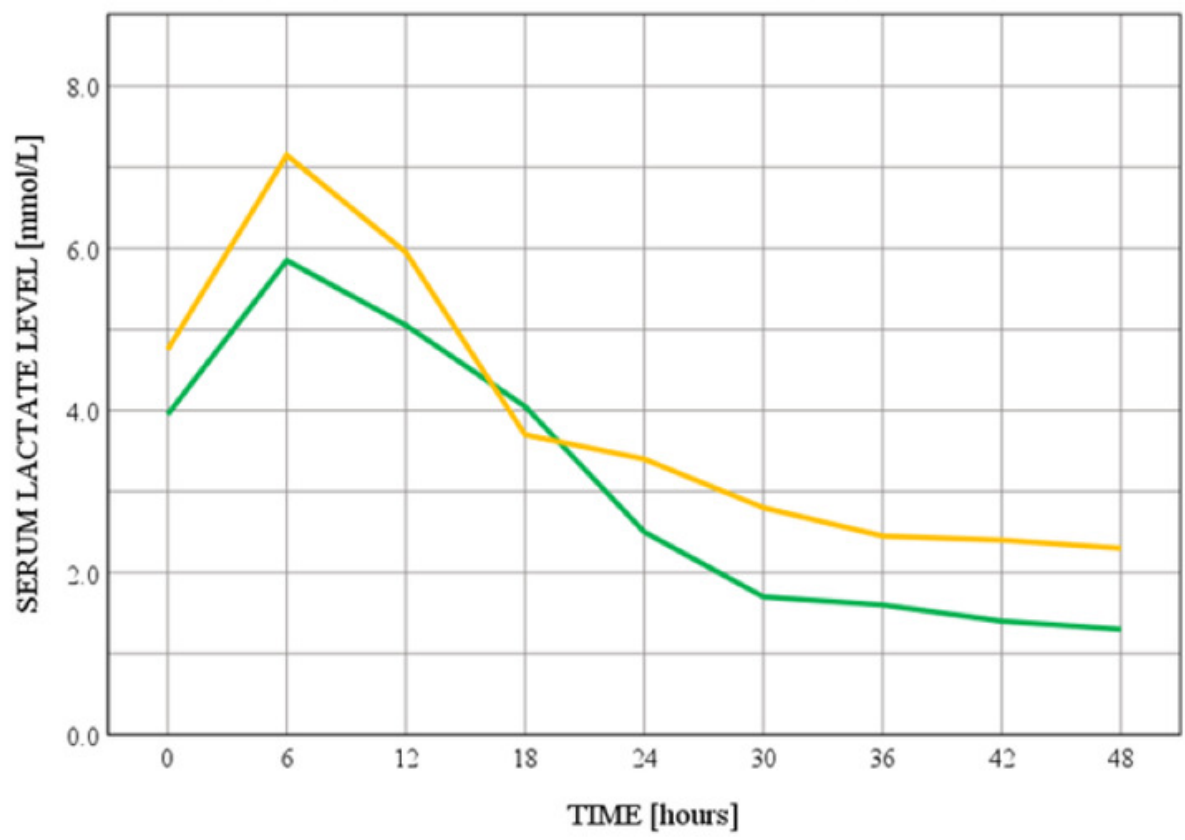

END OF THE STUDY SERUM LACTATE LEVEL (Lac 48) NORMAL - HYPERLACTATEMIA

\begin{tabular}{|c|l|c|}
\hline \multirow{2}{*}{ END OF THE STUDY } & $\begin{array}{c}\text { OBSERVATION } \\
\text { TIMI POINT }\end{array}$ & $\begin{array}{c}\text { SERUM LACTATE } \\
\text { LEVEL [mmol/L] }\end{array}$ \\
\hline \multirow{2}{*}{ NORMAL } & Lac 0 & $4.0(2.2-6.2)$ \\
\hline & Lac 48 & $1.3(1.1-1.5)$ \\
\hline & Peak Value & $6.2(3.9-8.3)$ \\
\hline \multirow{2}{*}{ HYPERLACTATEMIA } & Lac 0 & $4.8(2.5-6.6)$ \\
\hline & Lac 48 & $2.3(2.0-2.7)$ \\
\cline { 2 - 3 } & Peak Value & $7.6(4.5-9.0)$ \\
\hline
\end{tabular}




\section{Table $\mathbf{1}$ (on next page)}

Table 1. Baseline characteristics

Data shown as mean \pm SD or as median (IQR), or number (percentage). 


\begin{tabular}{|l|c|}
\hline VARIABLE & ANALYZED POPULATION; $\mathrm{n}=46$ \\
\hline Age, years & $48.7 \pm 11.7$ \\
\hline Male sex, $\mathrm{n}(\%)$ & $34(89.1)$ \\
\hline Dilated cardiomyopathy, n (\%) & $12(26.1)$ \\
\hline Ischemic cardiomyopathy, $\mathrm{n}(\%)$ & $16(34.8)$ \\
\hline Dyslipidemia, n (\%) & $21(45.7)$ \\
\hline Hypertension, $\mathrm{n}(\%)$ & $12(26.1)$ \\
\hline Diabetes, $\mathrm{n}(\%)$ & \\
\hline
\end{tabular}

1

2 Data shown as mean $\pm \mathrm{SD}$ or as median (IQR), or number (percentage).

3 Table 1. Baseline characteristics

4 